\title{
EDITORIAL
}

\section{LA DECLARACIÓN GATHER PARA LA PRESENTACIÓN PRECISA Y TRASPARENTE DE LOS ESTUDIOS SOBRE ESTIMACIONES DE SALUD PROCEDENTES DE MÚLTIPLES FUENTES DE INFORMACIÓN Y POBLACIONES}

\author{
Ferrán Catalá-López $(1,2,3)$ y Cristina Pérez Andrés (4).
}

(1) Departamento de Medicina, Universidad de Valencia/Instituto de Investigación Sanitaria INCLIVA y CIBERSAM. Valencia. España

(2) Fundación Instituto de Investigación en Servicios de Salud. Valencia. España

(3) Clinical Epidemiology Program. Ottawa Hospital Research Institute (OHRI). Ottawa. Ontario, Canada.

(4) Comité de Redacción de la Revista Española de Salud Pública.

La utilización de los indicadores de salud de la población puede servir para medir patrones epidemiológicos, valorar su estado de salud y la carga de las enfermedades, fijar prioridades sobre la asignación de recursos e identificar grupos poblacionales a los que deberían dirigirse las intervenciones y los programas de salud ${ }^{(1-6)}$. Clásicamente, la esperanza de vida al nacer, la mortalidad por todas las causas, la mortalidad infantil, la mortalidad materna, la prevalencia e incidencia de enfermedad han sido algunos de los principales indicadores empleados para medir la salud de las poblaciones e incluso para definir su grado de desarrollo social. En las últimas décadas se han propuesto otras medidas del tipo de las expectativas de salud (health expectancies) como la esperanza de vida libre de discapacidad, o del tipo de los diferenciales de salud (health gaps) como son los años de vida ajustados por discapacidad, que miden de manera conjunta las consecuencias mortales y no mortales de las enfermedades, las lesiones y los factores de riesgo a nivel poblacional ${ }^{(7)}$. Sin embargo, se reconoce que en numerosas ocasiones difícilmente se dispone de toda la información necesaria para generar las estimaciones más precisas y actualizadas por sexo, edad, espacio (geografía), tiempo (año) o problema de salud. Debido a las limitaciones de los propios datos, a menudo se utilizan diversas técnicas y modelos de simulación, más o menos complejos, que permiten establecer estimaciones integrando diversas fuentes de información (encuestas, estudios prospectivos, registros y sistemas de información) y asumiendo supuestos que no siempre se presentan con suficiente detalle. Para que las estimaciones obtenidas sean fiables y válidas para su correcta interpretación posterior y para la toma decisiones en políticas de salud pública, es necesaria la presentación clara, transparente y exacta de la información y de los métodos utilizados ${ }^{(8-10)}$.

En 2014, la Organización Mundial de la Salud (OMS) convocó un grupo de trabajo

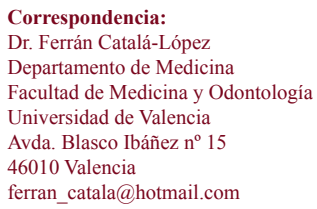

Cita sugerida: Catalá-López F, Pérez Andrés C. La Declaración GATHER para la presentación precisa y trasparente de los estudios sobre estimaciones de salud procedentes de múltiples fuentes de información y poblaciones Rev Esp Salud Pública. 2016;Vol. 90: 22 de agosto. e1-e3. 
con el objetivo de definir y promover buenas prácticas en la presentación de los resultados de las investigaciones sobre estimaciones de salud formado por dieciocho profesionales procedentes de la OMS, las Escuelas de Salud Pública de Harvard, Johns Hopkins y Londres, la Universidad de Oxford, la Universidad de Ottawa y la Universidad de Washington, entre otras instituciones, quienes revisaron las guías existentes para la presentación de resultados concluyendo que no garantizaban la de las estimaciones de salud global. Tras diferentes actividades, en febrero de 2015 se celebró una conferencia de consenso en Londres con el objetivo de acordar las directrices que se incluirían finalmente en la Declaración GATHER o Guidelines for Accurate and Transparent Health Estimates Reporting ${ }^{(9,10)}$, la cual tiene como objetivo mejorar la claridad y transparencia de la presentación de los resultados de las investigaciones que tienen por objetivo comparar el estado de salud de múltiples poblaciones y sus determinantes utilizando diferentes fuentes de información ${ }^{(9,10)}$. Las propuestas de la Declaración $\operatorname{GATHER}^{(9,10)}$ se recogen en forma de una lista de comprobación o checklist con 18 ítems que afectan a los cuatro apartados del texto de los artículos científicos que publican los resultados de estas investigaciones (Introducción, Métodos, Resultados y Discusión) y que se pueden resumir en:

-Presentar la fuente de financiación del trabajo y discutir potenciales conflictos de interés

- Definir los indicadores o medidas de salud, las poblaciones (incluyendo la edad, sexo y geografía) y los períodos de tiempo analizados

- Describir la identificación y el acceso a los datos

- Proporcionar las fuentes de información, sus características principales y el método de recolección de datos

- Proporcionar a descripción detallada del proceso de análisis y describir los métodos para el manejo de la incertidumbre de las estimaciones

- Indicar de qué manera puede accederse al código fuente utilizado para generar las estimaciones

- Presentar los resultados de las estimaciones de manera precisa y transparente

- Proporcionar acceso público de los datos y su análisis.

La publicación de la lista de comprobación de la Declaración GATHER viene acompañada de un documento de explicación y elaboración ${ }^{(11)}$ en el que se desarrolla el contenido de cada ítem y se proporcionan algunos ejemplos de publicación completa, adecuada y transparente de las fuentes de información.

Desde que fuera publicada en junio de 2016, la Declaración GATHER ${ }^{(9,10)}$ ha sido apoyada y adoptada por instituciones e iniciativas tan importantes como la Global Burden of Disease Study Collaboration a través del Institute for Health Metrics and Evaluation (IHME) de la Universidad de Washington $^{(12-14)}$, la red Enhancing the Quality and Transparency of Health Research (EQUATOR) ${ }^{(15)}$, la OMS ${ }^{(16)}$ así como por algunas revistas biomédicas con alto factor de impacto como The Lancet y PLOS Medicine, entre $\operatorname{otras}^{(17)}$.

Apoyar e implantar la Declaración GATHER $^{(9,10)}$ por parte de las revistas científicas que publican los resultados de las investigaciones del estado de salud de las poblaciones y por parte de quienes las financian y planifican así como de las autoridades sanitarias, puede contribuir a estimaciones más transparentes, completas y precisas que ayuden a mejorar las políticas de salud y la salud de la población.

La Revista Española de Salud Pública se suma a esta iniciativa incorporando los criterios de la Declaración GATHER en sus normas de publicación. 


\section{BIBLIOGRAFÍA}

1. Catalá López F, Alvarez Martín E, Gènova Maleras R, Morant Ginestar C. Relationship between research funding in the Spanish National Health System and the burden of disease. Rev Esp Salud Publica. 2009;83:137-51.

2. Gènova-Maleras R, Catalá-López F, de Larrea-Baz NF, Álvarez-Martín E, Morant-Ginestar C. The burden of premature mortality in Spain using standard expected years of life lost: a population-based study. BMC Public Health. 2011;11:787.

3. Gènova-Maleras R, Álvarez-Martín E, Morant-Ginestar C, Fernández de Larrea-Baz N, Catalá-López F. Measuring the burden of disease and injury in Spain using disability-adjusted life years: an updated and policy-oriented overview. Public Health. 2012;126:102431.

4. GBD 2013 Risk Factors Collaborators, Forouzanfar MH, Alexander L, Anderson HR, Bachman VF, Biryukov S, et al. Global, regional, and national comparative risk assessment of 79 behavioural, environmental and occupational, and metabolic risks or clusters of risks in 188 countries, 1990-2013: a systematic analysis for the Global Burden of Disease Study 2013. Lancet. 2015;386:2287-323.

5. GBD 2013 DALYs and HALE Collaborators, Murray CJ, Barber RM, Foreman KJ, Abbasoglu Ozgoren A, Abd-Allah F, Abera SF, et al. Global, regional, and national disability-adjusted life years (DALYs) for 306 diseases and injuries and healthy life expectancy (HALE) for 188 countries, 1990-2013: quantifying the epidemiological transition. Lancet. 2015;386:2145-91.

6. Global Burden of Disease Study 2013 Collaborators. Global, regional, and national incidence, prevalence, and years lived with disability for 301 acute and chronic diseases and injuries in 188 countries, 1990-2013: a systematic analysis for the Global Burden of Disease Study 2013. Lancet. 2015;386:743-800.

7. Murray CJ, Salomon JA, Mathers C. A critical examination of summary measures of population health. Bull World Health Organ. 2000;78(8):981-94.

8. Catalá-López F, Peiró S, Hutton B, Pérez Andrés C, Moher D. Declaration of transparency: promoting a more complete, honest and adequate publication of scientific articles. Rev Esp Salud Publica. 2014;88:1816.

9. Stevens GA, Alkema L, Black RE, Boerma JT, Collins GS, Ezzati $M$ et al. GATHER Working Group. Guidelines for Accurate and Transparent Health Estimates Reporting: the GATHER statement. PLoS Med. 2016 Jun 28;13(6):e1002056. eCollection 2016 Jun. Erratum in: PLoS Med. 2016 Aug;13(8):e1002116.
10. Stevens GA, Alkema L, Black RE, Boerma JT, Collins GS, Ezzati M, et al. Guidelines for Accurate and Transparent Health Estimates Reporting: the GATHER statement. Lancet. 2016 Jun 28. pii: S01406736(16)30388-9.

11. The GATHER Working Group. The GATHER statement: Explanation and Elaboration. Geneva: World Health Organization; 2016. Disponible en: http://gatherstatement.org/gather-statement/

12. Institute for Health Metrics and Evaluation. GATHER - A new age for global health metrics. Seattle: University of Washington; 2016. Disponible en: http:// www.healthdata.org/acting-data/gather-new-age-globalhealth-metrics

13. GBD 2015 HIV Collaborators, Wang H, Wolock TM, Carter A, Nguyen G, Kyu HH, et al. Estimates of global, regional, and national incidence, prevalence, and mortality of HIV, 1980-2015: the Global Burden of Disease Study 2015. Lancet HIV. 2016;3:e361-87.

14. GBD 2015 Mortality and Causes of Death Collaborators. Global, regional, and national life expectancy, all $\square$ cause and cause specific mortality for 249 causes of death, 1980-2015: a systematic analysis for the Global Burden of Disease Study 2015. Lancet. 2016 (en prensa).

15. The EQUATOR network. GATHER statement. Accesible en: http://www.equator-network.org/reportingguidelines/gather-statement/

16. Stevens GA, Hogan DR, Boerma T. Improving reporting of health estimates. Bull World Health Organ. 2016;94:483.

17. The GATHER Working Group. The GATHER statement: Endorsement. Disponible en: http://gather-statement.org/endorsement/ 